\title{
Mudanças Curriculares no Ensino Médico Brasileiro: um Debate Crucial no Contexto do Promed
}

\section{PALAVRAS-CHAVE}

- Educação médica;

- Currículo médico;

- Estudantes de medicina;

- Diretizes Curriculares Nacionais;

- Promed;

- Sistema Único de Saúde.

\section{KEY WORDS}

- Medical education;

- Medical curriculum;

- Medical students;

- National curricular guidelines;

- Promed;

- Brazilian Health System.

Recebido em: 28/11/2007

Aprovado em: 13/01/2008

REVISTA BRASILEIRA DE EDUCAÇÃO MÉDICA

\section{Curricular Changes in Brazilian Medical Education: a Crucial Discussion in the Context of Promed}

Neilton Araújo de Oliveira Rosane Moreira Silva de Meirelles ${ }^{I I}$ Geraldo Cunha Cury III Luiz Anastácio Alves ${ }^{I I}$

\begin{abstract}
RESUMO
A educação médica passa por modificações na doutrina e na prática da formação profissional, conectada à contemporaneidade do mundo globalizado. No contexto do Sistema Único de Saúde (SUS), aumenta o interesse de diferentes sujeitos em relação ao ensino médico, devido aos aspectos políticos e comunitários e com repercussões nas mudanças nos serviços de saúde. Iniciativas de incentivo às mudanças curriculares em medicina são adotadas para incrementar melhorias na formação médica. Nesse contexto se insere o Projeto de Incentivo a Mudanças Curriculares para os Cursos de Medicina (Promed). Com o objetivo de analisar a percepção de alunos sobre mudanças curriculares na educação médica, pesquisamos seis cursos médicos, em três estados brasileiros, usando questionários e entrevistas. Alguns pressupostos das Diretrizes Curriculares Nacionais não foram incrementados, mas o desdobramento do Promed possibilitou um programa ampliado de incentivos a mudanças curriculares. Mesmo tendo caráter exploratório, este estudo aponta a necessidade de estudos prospectivos para conhecer os impactos dos incentivos às mudanças curriculares do ensino médico, sintonizando-o, assim, com as necessidades de saúde da população.
\end{abstract}

ABSTRACT
Medical Education is undergoing modifications in the doctrine and in the practice of professional edu-
cation as a consequence of today's globalized world. With respect to the Brazilian Health System, dif-
ferent subjects show increasing interest in medical education and in changes in the healthcare services.
There are initiatives for encouraging changes in the medical curriculum for improving medical educa-
tion. The Program for the Encouragement of Curricular Changes in Medical Courses (Promed) was
implemented in this context. In order to analyze the opinion of students about changes in the curricu-
lum of the medical course, we studied six medical schools in three Brazilian states, using questionnaires
and interviews. Some of the propositions of the National Curriculum Guidelines have not been met
but Promed gave rise to a new extensive program of changes in the medical curriculum. Even having
exploratory character, this work clearly indicates the need for prospective studies in order to know the
impact of Promed on medical education for adapting it to the healthcare needs of the population.

${ }^{1}$ Universidade Federal do Tocantins, Tocantins, Brasil; Fundação Oswaldo Cruz, Rio de Janeiro, Brasil.

${ }^{\text {II }}$ Fundação Oswaldo Cruz, Rio de Janeiro, Brasil

III Universidade Federal de Minas Gerais, Minas Gerais, Brasil. 


\section{INTRODUÇÃO}

A educação médica passa por modificações de toda ordem, na doutrina e na prática da formação profissional conectada à contemporaneidade de um mundo globalizado ${ }^{1} \mathrm{e}$ condicionada por parâmetros biologicistas, humanísticos e éticos, mas ultimamente também pelos aspectos políticos e comunitários relacionados às mudanças nos sistemas e serviços de saúde, na implementação do Sistema Único de Saúde (SUS)2 ${ }^{2}$ O interesse pela transformação e mudanças na educação médica vem aumentando nas duas últimas décadas ${ }^{3,4} \mathrm{com}$ o envolvimento de educadores, pesquisadores, gestores, estudantes, profissionais e entidades da área - como o Conselho Federal de Medicina (CFM) e a Associação Brasileira de Educação Médica (Abem) - além do Ministério da Saúde (MS) e do Ministério da Educação (MEC), à medida que cresceu a percepção de que sem profissionais formados com um novo perfil se tornava muito difícil a tarefa de reorganizar modelos de atenção à saúde, conforme preconizado pelo SUS, identificados com os princípios de integralidade, humanização, qualidade da atenção, etc. ${ }^{2,5}$.

Também no âmbito internacional este tema tem merecido maior atenção ${ }^{6-9}$. Eventos como a Segunda Conferência de Edimburgo, o Encontro Internacional de Educação Médica ${ }^{10} \mathrm{e}$ o programa da OMS voltado para a transformação da educação médica ${ }^{11}$ são exemplos desse interesse.

A adequação da formação médica à estratégia de "atenção primária de saúde" já vinha sendo discutida a partir da Conferência de Alma Ata ${ }^{12}$ e se intensificou no processo de construção do SUS. Isto tem levado a questionamentos, proposições e inúmeros debates sobre ensino médico, em congressos de educação médica ${ }^{13}$, encontros de gestores ${ }^{14}$, reuniões, seminários, conferências nacionais de saúde ${ }^{15}$ e em múltiplas teses ${ }^{16}$, além de revistas de educação médica.

A ausência de uma política de recursos humanos para a saúde, formulada e realizada sob a lógica da política pública expressa nos princípios e diretrizes constitucionais da Seguridade Social e do SUS ${ }^{17}$, compõe hoje, com a escassez de recursos, os dois maiores obstáculos ao desenvolvimento do SUS, a despeito de a educação na área de saúde nunca ter sido tão enfatizada. Não se trata mais de formar pessoal competente tecnicamente, mas profissionais que tenham vivência sobre o acesso universal, a qualidade e humanização na Atenção à Saúde, com Controle Social, o que significa dizer integração efetiva e permanente entre formação médica e serviços de saúde ${ }^{18,19}$.

De acordo com um estudo Comissão Interinstitucional Nacional de Avaliação do Ensino Médico (Cinaem), os recém-graduados em medicina no Brasil terminam o curso com apenas metade dos conhecimentos que deveriam ter, e a residência médica passou a ser a continuidade natural da graduação ${ }^{20}$. A quase totalidade dos médicos que se formam ingressa na residência médica a fim de obter especialização, sendo que, com a introdução do Programa Saúde da Família, houve uma mudança no sistema de atenção básica e isso exige uma formação profissional adequada para essa nova realidade ${ }^{20}$.

Mais recentemente, a partir de 2001, os ministérios da Saúde e da Educação têm formulado políticas destinadas a promover mudanças na formação e na distribuição geográfica dos profissionais de saúde: Diretrizes Curriculares Nacionais dos Cursos da Área de Saúde ${ }^{21}$; Programa de Incentivo às Mudanças Curriculares nos Cursos de Medicina (Promed) ${ }^{22}$; VER-SUS, uma estratégia de vivência no SUS para estudantes dos cursos de saúde; Programa de Interiorização do Trabalho em Saúde (PITS ${ }^{17}$; Pólos de Educação Permanente do SUS; e, já no final de 2005, o Programa Nacional de Reorientação da Formação Profissional em Saúde (Pró-Saúde) ${ }^{23}$.

A Quadro 1 sintetiza os principais objetivos e características desses programas e projetos. Os processos de reformulação curricular, no Brasil e em outros países, têm obedecido a diferentes diretrizes, tentando conciliar tendências pedagógicas e políticas ${ }^{24}$.

O Ministério da Saúde tem a responsabilidade, por definição constitucional e da Lei 8.080/90 — Lei Orgânica da Saúde ${ }^{25}$ - , de estimular e ordenar o processo de formação de trabalhadores em saúde. Para formar profissionais com o perfil que atenda às necessidades do SUS, os cursos de saúde (incluindo os de medicina) precisam adequar sua abordagem pedagógica, favorecer a articulação dos conhecimentos e promover atividades práticas ao longo de todo o curso em todos os tipos de unidades de saúde.

Em 2002, as escolas médicas brasileiras ofereciam 10.101 vagas anuais, das quais mais da metade $(57,4 \%)$ estão na $\operatorname{Re}-$ gião Sudeste. A relação profissional/habitantes (1,6 médico / 1.000 habitantes ou 1 médico / 622 habitantes), no âmbito nacional, está de acordo com os padrões da Organização Mundial de Saúde (OMS). No entanto, ao se analisar a distribuição geográfica dos profissionais (Tabela 1), verifica-se muita desigualdade na distribuição por região e dentro de cada estado, na localização capital e interior ${ }^{17}$. Apesar da abertura de muitas novas escolas médicas a partir de 2000, a região com o pior índice (1 médico por 1.345 habitantes) é a Região Norte ${ }^{18}$, mesmo considerando ser esta a região mais dependente do SUS ${ }^{19}$ e onde mais se expandiu o Saúde da Família nos últimos cinco anos.

À distribuição desigual da população nas diferentes regiões brasileiras segue-se uma também desigual distribuição de 


\section{QUADRO 1}

Principais objetivos e características dos projetos / programas de incentivos a mudanças na educação médica no Brasil — 2001-2005

\begin{tabular}{|c|c|c|}
\hline $\begin{array}{l}\text { Projeto/ } \\
\text { Programa }\end{array}$ & Público & Objetivos - Características \\
\hline $\begin{array}{l}\text { Diretrizes } \\
\text { Curriculares } \\
\text { Nacionais dos } \\
\text { Cursos da Saúde }\end{array}$ & $\begin{array}{l}\text { Os } 14 \text { cursos da } \\
\text { área da Saúde }\end{array}$ & $\begin{array}{l}\text { - Articulação entre educação superior e sistemas de saúde, objetivando formação geral e específica } \\
\text { dos egressos / profissionais, com ênfase na promoção, prevenção, recuperação e reabilitação da saúde, } \\
\text { indicando competências comuns gerais para esse perfil de formação. } \\
\text { - Conceitos de saúde, princípios e diretrizes do SUS como elementos fundamentais dessa articulação. } \\
\text { - Indicar tópicos ou campos de estudos de ensino-aprendizagem, com ampla liberdade para a } \\
\text { integralização curricular. } \\
\text { - Levar os alunos dos cursos da saúde a aprender a aprender, o que engloba aprender a ser, aprender a } \\
\text { fazer, aprender a viver juntos e aprender a conhecer. } \\
\text { - Construir perfil acadêmico e profissional com competências, habilidades e conteúdos } \\
\text { contemporâneos. } \\
\text { - Capacitar alunos / profissionais para atuarem com qualidade e resolutividade no SUS. }\end{array}$ \\
\hline $\begin{array}{l}\text { Promed } \\
\text { - Programa } \\
\text { de Incentivo } \\
\text { às Mudanças } \\
\text { Curriculares nos } \\
\text { Cursos de Medicina }\end{array}$ & $\begin{array}{l}\text { Escolas e Cursos } \\
\text { de graduação } \\
\text { em Medicina }\end{array}$ & $\begin{array}{l}\text { - Oferecer cooperação técnica e/ou operacional às escolas de graduação em Medicina que se } \\
\text { dispuseram a adotar processos de mudança nos currículos de seus cursos, com enfoque para as } \\
\text { necessidades de saúde da população e do Sistema Único de Saúde. } \\
\text { - Programar estágios nos hospitais universitários e em toda a rede de serviços e atividades extraclasse } \\
\text { contemplando os principais problemas de saúde da população. } \\
\text {-Capacitar melhor os estudantes de medicina para atender aos principais problemas de saúde da } \\
\text { população, de acordo com a nova realidade de funcionamento do SUS, sendo necessário, para isso, } \\
\text { que os cursos de medicina possibilitassem a formação de médicos com competência geral, essencial à } \\
\text { ampliação de programas de atenção básica, como o Programa Saúde da Família (PSF). }\end{array}$ \\
\hline VER-SUS & $\begin{array}{l}\text { Alunos de } \\
\text { graduação de } \\
\text { vários cursos da } \\
\text { área de saúde }\end{array}$ & $\begin{array}{l}\text { - Aproximação da política de saúde com a formação acadêmica, em que estudantes de graduação } \\
\text { vão conhecer de perto, em cidades de todas as regiões do País, o funcionamento do Sistema Único de } \\
\text { Saúde de maneira integral — gestão do setor, organização e práticas de atenção à saúde, formação e } \\
\text { desenvolvimento profissional, participação popular e controle social. } \\
\text { - Familiarização com o SUS, seus problemas, peculiaridades e avanços. } \\
\text { - Participação ativa na direção das diferentes entidades estudantis da área da saúde na construção } \\
\text { da vivência, desde seu desenho e negociação com os municípios até o processo de inscrição dos } \\
\text { estudantes. }\end{array}$ \\
\hline $\begin{array}{l}\text { PITS - Programa } \\
\text { de Interiorização } \\
\text { dos Trabalhadores } \\
\text { em Saúde }\end{array}$ & $\begin{array}{l}\text { Profissionais } \\
\text { de medicina e } \\
\text { enfermagem }\end{array}$ & $\begin{array}{l}\text { - Incentivo técnico, pedagógico e financeiro para fixação de médicos e enfermeiros no Programa } \\
\text { Saúde da Família em pequenos municípios brasileiros, onde não existe médico. } \\
\text { - Interiorização de profissionais de saúde. } \\
\text { - Articulação de instituições de ensino e serviços de saúde, para acompanhamento e capacitação } \\
\text { desses profissionais. }\end{array}$ \\
\hline $\begin{array}{l}\text { Pólos de Educação } \\
\text { Permanente - SUS }\end{array}$ & & $\begin{array}{l}\text { - Articulação interinstitucional, em um território, entre gestores federais, estaduais e municipais do } \\
\text { SUS, universidades e instituições de ensino com cursos na área da saúde, incluindo principalmente } \\
\text { suas áreas clínicas e de saúde coletiva; centros formadores, escolas de saúde pública, núcleos de } \\
\text { saúde coletiva, hospitais universitários; estudantes da área de saúde, trabalhadores de saúde; } \\
\text { Conselhos Municipais e Estaduais de Saúde; movimentos estudantis e sociais que trabalham com } \\
\text { saúde, fortalecendo compromissos com o SUS. } \\
\text { - Discutir e implementar projetos de mudança do ensino formal e da educação permanente dos } \\
\text { trabalhadores de saúde. } \\
\text { - Propõe a adoção da educação permanente como a estratégia fundamental para a recomposição das } \\
\text { práticas de formação, atenção, gestão, formulação de políticas e controle social no setor da saúde. } \\
\text { - Propõe ainda que formação e desenvolvimento devem feitos de modo descentralizado, ascendente, } \\
\text { transdisciplinar e devem propiciar a democratização institucional. }\end{array}$ \\
\hline Pró-Saúde & $\begin{array}{l}\text { Escolas e cursos } \\
\text { de medicina, } \\
\text { enfermagem e } \\
\text { odontologia }\end{array}$ & $\begin{array}{l}\text { - Incentivar transformações de processo de formação, geração de conhecimentos e prestação } \\
\text { de serviços de saúde à população, para abordagem integral do processo de saúde-doença. } \\
\text { Reorientar o processo de formação em medicina, enfermagem e odontologia, de modo a oferecer } \\
\text { à sociedade profissionais habilitados para responder às necessidades da população brasileira e à } \\
\text { operacionalização do SUS. } \\
\text { - Aproximação entre a formação de graduação em saúde no País e as necessidades da atenção básica } \\
\text { à saúde, que se traduzem, no Brasil, pela estratégia de Saúde da Família. } \\
\text { - Estabelecer mecanismos de cooperação entre os gestores do SUS e as escolas de medicina, } \\
\text { enfermagem e odontologia, com vistas à melhoria da qualidade e resolutividade da atenção à saúde } \\
\text { prestada ao cidadão, bem como à integração da rede pública de serviços de saúde e à formação dos } \\
\text { profissionais na graduação e na educaço permanente. }\end{array}$ \\
\hline
\end{tabular}

Nota: Tabela preparada com dados e informações das fontes: MS (2005/2006) e MEC (2005/2006). 
FIGURA 1

Distribuição percentual de cursos médicos — grandes regiões — Brasil, 2005.

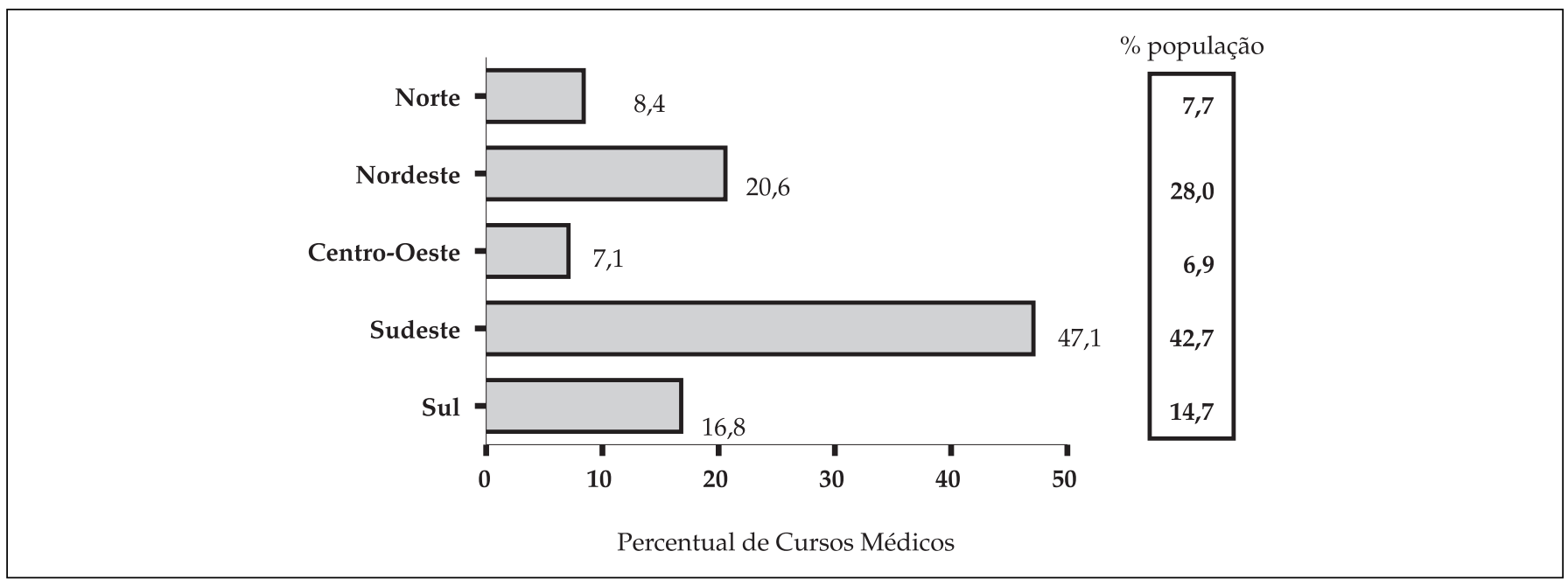

Preparado a partir de dados do MEC/Inep e IBGE 2005.

cursos médicos no País (Figura 1). Contudo, apesar de constituir um aspecto que tem sido discutido, de forma relevante, por diferentes instituições das áreas da educação e da saúde, essa desproporção pouco implicou mudanças dessas políticas, persistindo a concentração das escolas médicas nos grandes centros urbanos, sobretudo nas regiões Sul e Sudeste.

Entre os diversos aspectos relativos à inadequação da formação médica, possivelmente o fator mais agravante diz respeito ao perfil do médico formado: centrado no modelo biologicista e na medicalização, que tem no hospital o centro de toda a formação médica; por conseguinte, a atuação profissional futura estará muito em desacordo com as necessidades de saúde da população ${ }^{26}$.

\section{MUDANÇAS PROPOSTAS PELO PROMED}

Após diversos debates durante os anos de 2001 e 2002, inclusive em audiências públicas nos Conselhos Nacionais de Saúde e Educação, o MS, Opas e MEC, em parceria com a Abem e a Rede Unida, elaboraram o Promed, uma cooperação técnica patrocinada pelo Ministério da Saúde para as escolas de graduação em medicina que se dispuseram a adotar processos de mudança nos currículos de seus cursos, com enfoque nas necessidades de saúde da população e do SUS. O Termo de Referência do Programa, cujo lema era "uma nova escola médica para um novo sistema de saúde", explicita três eixos para o desenvolvimento das mudanças: orientação teórica, abordagem pedagógica e cenários de prática ${ }^{22}$.

TABELA 1

Relação médico/habitantes e percentuais de médicos ativos e de população (capital e interior) por grande região — Brasil, maio, de 2003

\begin{tabular}{lccccccc}
\hline & \multirow{2}{*}{ Região } & Relação & \multicolumn{3}{c}{$\%$ - Médicos Ativos } & \multicolumn{3}{c}{$\%$ População } \\
& Méd/Hab & Total & Capital & Interior & Total & Capital & Interior \\
\hline Norte & $1 / 1.345$ & 3,6 & 62,1 & 37,9 & 7,7 & 30 & 70 \\
Nordeste & $1 / 1.063$ & 16,4 & 66,8 & 31,1 & 28,0 & 21 & 79 \\
Centro-Oeste & $1 / 640$ & 6,7 & 68,9 & 31,1 & 6,9 & 37 & 63 \\
Sudeste & $1 / 640$ & 58,4 & 46,4 & 53,6 & 42,7 & 26 & 74 \\
Sul & $1 / 455$ & 14,9 & 35,9 & 64,1 & 14,7 & 13 & 87 \\
Brasil & $1 / 622$ & 100 & 50,1 & 49,9 & 100 & 20 & 80 \\
\hline
\end{tabular}

Tabela preparada a partir de dados das fontes IBGE (2003) e CFM (2003). 
QUADRO 2

Escolas médicas selecionadas para participarem do Promed, 2002

\begin{tabular}{|l|l|l|}
\hline \multicolumn{1}{|c|}{ Universidade Federal } & \multicolumn{1}{|c|}{ Instituição Estadual / Municipal } & \multicolumn{1}{c|}{ Instituição Privada } \\
\hline Goiás (UFG) & Fundação Universidade de Pernambuco (UPE) & Pontifícia Universidade Católica de São Paulo (PUC-SP) \\
\hline Pernambuco (UFPE) & Univ. de Ribeirão Preto (Unaerp) & $\begin{array}{l}\text { Pontifícia Universidade Católica do Rio Grande do Sul } \\
\text { (PUC-RS) }\end{array}$ \\
\hline Ceará (UFC) & Univ. Estadual de Campinas (Unicamp) & \\
\hline Roraima (UFRR) & Univ. Estadual Paulista Júlio Mesquita Filho (Unesp) & \\
\hline São Paulo (USP) & Univ. Estadual de Montes Claros (Unimontes) & \\
\hline Minas Gerais (UFMG) & Fundação Educ. Serra dos Órgãos (Feso) & \\
\hline Fluminense (UFF) & Univ. Estadual de Londrina (UEL) & \\
\hline Rio Grande do Sul (UFRGS) & & \\
\hline Santa Catarina (UFSC) & & \\
\hline
\end{tabular}

Nota: Tabela preparada a partir de informações do MS (Agência Saúde/MS) — 25/11/2002.

Das 92 faculdades de medicina que o Brasil tinha em 2002 — formando por ano 7.500 médicos —, 55 apresentaram propostas de mudanças curriculares na primeira etapa de seleção. A escolha final das 20 escolas (Quadro 2) foi feita por uma comissão composta por docentes da educação médica, representantes dos Conselhos de Secretários Estaduais (Conass) e de Secretários Municipais de Saúde (Conasems) ${ }^{22}$.

Tratando-se de um projeto de adesão, a grande participação das universidades significava o interesse que elas demonstravam na proposta de mudanças. Em fins de novembro de 2002, 19 das 20 escolas de medicina selecionadas pelo Promed oficializaram suas propostas e passaram a receber, a partir de 2003, os recursos financeiros para o desenvolvimento das atividades ${ }^{22}$.

Até porque não há o entendimento de que todos os serviços de saúde - e não só os hospitais universitários — são e devem ser locais de ensino ${ }^{26}$, o principal objetivo dos incentivos era oferecer cooperação técnica e/ou operacional para as escolas de graduação em medicina. A condição para isso era que as escolas deveriam se dispor a adotar processos de mudança nos currículos de seus cursos, com enfoque nas necessidades de saúde da população e do Sistema Único de Saúde. O projeto também previa a adoção de metodologias ativas de ensino-aprendizagem e formação geral, crítica e humanística, além de abrir possibilidades de capacitação em torno de temas importantes para as mudanças, como clínica ampliada de saúde e forte integração ensino-serviços de saúde ${ }^{22}$.

Diante do debate em torno dos inúmeros fatores e das diferentes iniciativas que buscam contribuir, no contexto do SUS, para o processo de mudança no ensino médico nos últimos anos no Brasil, apresentamos aqui um estudo exploratório da percepção de alunos de medicina sobre mudanças, sintonizadas com as Diretrizes Curriculares Nacionais ${ }^{21}$, especialmente a partir do Promed. Ressaltamos que o Promed não é o único dispositivo a implementar mudanças curriculares, nem é nossa pretensão avaliar a efetividade do mesmo. Como, também, buscamos conhecer a compreensão que outros sujeitos - incluindo gestores de serviços de saúde e coordenadores desse projeto - têm a respeito do desenvolvimento do Promed e suas repercussões no processo de mudanças curriculares, entendemos que o presente trabalho poderá representar uma oportuna reflexão em torno dessas mudanças e uma contribuição relevante para a pertinência desse debate.

\section{METODOLOGIA}

Entre 2006 e 2007, foram pesquisados seis cursos médicos de três estados de diferentes regiões brasileiras — sendo dois cursos participantes e quatro não participantes do programa —, na tentativa de um estudo piloto de análise das mudanças curriculares relativas às Diretrizes Curriculares Nacionais a partir do Promed. Com levantamento bibliográfico nos sites do Ministério da Saúde (MS), Ministério da Educação (MEC), Conselho Federal de Medicina (CFM), Pubmed, Scielo, revistas da área de educação e consulta ao banco de teses da Capes, definiram-se os enfoques dessa análise.

A partir das questões centrais da mudança curricular e das principais dificuldades apontadas em oficinas nacionais de avaliação pelos cursos de medicina envolvidos no Promed, foi aplicado um questionário a 318 estudantes do internato de seis escolas médicas, selecionadas em três estados brasileiros. Os alunos, em sua maioria, freqüentavam o último ano do curso médico, sendo 159 alunos de cursos Promed e 159 de cursos que não aderiram ao programa. Nos seis cursos médicos estudados, os concluintes totalizavam 724 alunos, tendo-se obtido 43,92\% (318 alunos) de questionários respondidos. Nos dois cursos Promed, do total de 198 concluintes, foram obtidos 159 questionários respondidos (80,30\%), enquanto nos cursos 
não Promed, do total de 526 concluintes, 159 responderam ao questionário, representando $30,23 \%$ dos alunos destes cursos.

Na seqüência, verificamos as respostas à nossa pesquisa nesses dois grupos de alunos e sua pertinência ao debate em torno da adoção das Diretrizes Curriculares Nacionais. As respostas foram analisadas em sete categorias: processos de mudanças curriculares; concepção e enfoque pedagógico; estrutura e condições materiais do curso; programas e/ou atividades de pesquisas científicas; integração ensino-serviços de saúde-comunidade; resultado final da formação médica; avaliação e regulação do ensino médico. Na análise dos dados quantitativos foi realizada a distribuição da freqüência absoluta e percentual dos parâmetros pesquisados.

Para melhor compreender esses achados, foi realizada uma entrevista semi-estruturada com gestores de escolas médicas, docentes, alunos, gestores de serviços de saúde, consultores, além de componentes da direção e coordenação do programa, com pelo menos um representante de cada um desses grupos, num total de 23 sujeitos, todos participantes do Promed, em busca de indícios das principais dificuldades encontradas pelas instituições envolvidas nesse programa.

A observação participante foi utilizada como mais uma via de acesso à realidade investigada, refletindo a riqueza metodológica que é articular as falas dos sujeitos com as situações concretas em que vivem ${ }^{27}$. Entretanto, diante da existência de vários aspectos e um substrato comum de identidade entre nossa prática cotidiana de trabalho e esta nossa investigação, o que torna essa relação "solidariamente imbricada e comprometida, sentimo-nos impelidos a fazer uma advertência preventiva, repetindo Lévi-Strauss (1975): Numa ciência onde o observador é da mesma natureza que o objeto, o observador, ele mesmo, é uma parte de sua observação"28.

Muitas das entrevistas foram realizadas por ocasião de duas das oficinas de avaliação do Promed durante encontros e congressos da Abem, tendo como foco os diferentes parâmetros propostos pelas Diretrizes Curriculares Nacionais. Por conseguinte, estes foram os critérios para seleção das falas de diferentes sujeitos escolhidas para ilustrar nossa reflexão. Entre as diversas variáveis estudadas nas entrevistas, as respostas dos sujeitos foram submetidas a uma análise de conteúdo ${ }^{29}$. Foram priorizadas aquelas mais relacionadas à integração entre ensino, serviços de saúde e participação comunitária.

Todos os sujeitos que participaram do estudo assinaram um termo de consentimento livre e esclarecido, e o projeto de pesquisa obteve aprovação do Comitê de Ética e Pesquisa do IOC - Fiocruz (Parecer 302-06).

\section{RESULTADOS}

Os dados socioeconômicos dos alunos pesquisados $(\mathrm{N}=$ 318) são mostrados na Tabela 2. Mais de $50 \%$ dos alunos, em todos os cursos, apresentam idade entre 23 e 25 anos. Em relação ao sexo, em dois dos seis cursos predominam as mulheres. Quanto ao estado civil, um curso apresenta 78\% de estudantes solteiros, enquanto outro curso tem $84 \%$, e os demais apresentam $89 \%$ ou mais de alunos solteiros. Sobre o item cor, autodefinida por cada um dos respondentes, somados os valores das respostas "branca" e "parda" - por serem as usualmente predominantes na realidade brasileira - , nota-se que cerca de $90 \%$ dos alunos se enquadram nesta categoria. No quesito renda familiar mensal, exceto em um dos cursos, em todos os demais os alunos apresentam $80 \%$ de respostas referindo renda familiar mensal com mais de 15 salários mínimos, e mais da metade dos alunos refere renda em média acima de 20 salários mínimos. Os alunos cujos pais têm curso superior e/ou pósgraduação superam $60 \%$ em todos os cursos; em três cursos, esse percentual chega a mais de $70 \%$. Em metade dos cursos, de $69 \%$ a $75 \%$ dos alunos possuem computador; na outra metade dos cursos, esse percentual ultrapassa $90 \%$.

Os resultados obtidos, destacando-se diferentes aspectos, estão sintetizados na Tabela 3.

Era de se esperar maior percentual de respostas positivas nos cursos Promed quanto à questão se o curso adota as Diretrizes Curriculares Nacionais, porém se observa o contrário: 40,2\% de alunos Promed e 60,3\% de alunos não Promed dizem que seu curso as adota, pelo menos em parte. Isto pode significar que estes cursos possivelmente implantaram outros programas de mudanças, confirmando que, além do Promed, outras iniciativas de mudanças identificadas com as Diretrizes Nacionais Curriculares estão ocorrendo em cursos de graduação da medicina. $\mathrm{O}$ fato de um curso ser de uma universidade nova, por exemplo - como é o caso de dois dos cursos não Promed pesquisados —, seguramente terá importância, uma vez que, provavelmente, ali foi adotado um currículo já identificado com as Diretrizes Nacionais Curriculares.

Segundo os alunos, somente em cerca de $13 \%$ nos cursos Promed e em torno de $20 \%$ nos cursos não Promed se discute a política de saúde durante todo o curso, desde o primeiro ano. A predominância de alunos que pretendem ser especialistas, ao contrário do que se esperava, é maior em cursos Promed $(75,9 \%)$ do que em cursos não Promed (68,7\%). Só uma minoria - cerca de um quarto dos alunos - tanto dos cursos Promed como dos cursos não Promed - pretende trabalhar em Saúde Pública. Por outro lado, 63,5\% dos alunos Promed e 65,4\% dos alunos não Promed tendem a escolher, concomitantemente, vínculos públicos e privados para atuar quando formados. 
$\mathrm{Na}$ análise das respostas dos alunos, constatou-se, nas escolas pesquisadas, que os estudantes apresentam opiniões bastante diversas, tanto na comparação de cursos Promed com cursos não participantes do Promed, quanto na comparação de cursos participantes do Promed entre si, ou não participantes do Promed entre si. Assim, pode-se pensar que muitos outros fatores contribuem para influenciar o processo de mudanças na educação médica e para definir o perfil e a qualificação do médico.

Para conhecer mais o que pensavam os sujeitos envolvidos no Promed e avaliar melhor alguns pontos verificados na pesquisa quantitativa, nas entrevistas com alguns deles, quando se perguntou aos alunos "você já decidiu onde trabalhar, depois de formado? E por quê?", a resposta de um desses alunos foi:
Olha, hoje é muito difícil começar uma carreira... quando entrei na faculdade não sabia direito o que fazer, mas já sei que quero ser sanitarista. Eu quero estar inserido no SUS também para fazer uma medicina mais ampla, socializada.

Ao mesmo tempo, outro estudante respondeu:

A gente tem pouca orientação, durante o nosso curso, sobre as condições para exercer a medicina (...) o médico virou um assalariado e com poucas chances de escolher; qualquer lugar hoje é muito complicado e o que sobra é quase só serviço público ou atender plano de saúde.

TABELA 2

Perfil socioeconômico dos alunos (6ª ano médico) pesquisados (\%), Brasil, 2006-2007

\begin{tabular}{|c|c|c|c|c|c|c|c|c|}
\hline \multirow[t]{2}{*}{ Item } & \multicolumn{2}{|c|}{ Parâmetro } & $\begin{array}{l}\text { Curso } 1 \\
N=72\end{array}$ & $\begin{array}{c}\text { Curso } 2 \\
N=24\end{array}$ & $\begin{array}{c}\text { Curso } 3\left(^{*}\right) \\
N=91\end{array}$ & $\begin{array}{l}\text { Curso } 4 \\
N=27\end{array}$ & $\begin{array}{l}\text { Curso } 5 \\
N=36\end{array}$ & $\begin{array}{c}\text { Curso } 6\left(^{*}\right) \\
N=68\end{array}$ \\
\hline & \multicolumn{2}{|c|}{20 a 22} & 8 & 8 & 9 & 15 & - & 7 \\
\hline \multirow[t]{2}{*}{ Idade (anos) } & \multicolumn{2}{|c|}{23 a 25} & 67 & 63 & 59 & 52 & 69 & 62 \\
\hline & \multicolumn{2}{|c|}{+ de 25} & 25 & 29 & 32 & 33 & 31 & 31 \\
\hline \multirow{3}{*}{ Sexo } & \multicolumn{2}{|c|}{ Masc. } & 42 & 54 & 54 & 44 & 58 & 51 \\
\hline & \multicolumn{2}{|c|}{ Fem. } & 58 & 46 & 46 & 56 & 42 & 49 \\
\hline & \multicolumn{2}{|c|}{ Solt. } & 92 & 92 & 84 & 78 & 89 & 90 \\
\hline \multirow[t]{2}{*}{ Estado civil } & \multicolumn{2}{|c|}{ Casado } & 8 & 8 & 15 & 22 & 11 & 9 \\
\hline & \multicolumn{2}{|c|}{ Outro } & 0 & 0 & 1 & 0 & 0 & 1 \\
\hline \multirow{5}{*}{ Cor } & \multicolumn{2}{|c|}{ Negra } & 0 & 0 & 4 & 11 & 3 & 4 \\
\hline & \multicolumn{2}{|c|}{ Parda } & 27 & 25 & 48 & 30 & 22 & 44 \\
\hline & \multicolumn{2}{|c|}{ Amarela } & 3 & 0 & 3 & 0 & 3 & 3 \\
\hline & \multicolumn{2}{|c|}{ Branca } & 70 & 71 & 44 & 59 & 67 & 49 \\
\hline & \multicolumn{2}{|c|}{ Outra } & 0 & 4 & 1 & 0 & 5 & 0 \\
\hline \multirow{5}{*}{$\begin{array}{l}\text { Renda familiar } \\
\text { mensal (em salários } \\
\text { mínimos) }\end{array}$} & \multicolumn{2}{|c|}{ Até 5} & 0 & 4 & 0 & 4 & 0 & 0 \\
\hline & \multicolumn{2}{|c|}{+ de 5 a 10} & 3 & 17 & 7 & 4 & 3 & 1 \\
\hline & \multicolumn{2}{|c|}{+ de 10 a 15} & 4 & 29 & 11 & 15 & 6 & 9 \\
\hline & \multicolumn{2}{|c|}{+ de 15 a 20} & 17 & 21 & 28 & 18 & 22 & 40 \\
\hline & \multicolumn{2}{|c|}{+ de 20} & 76 & 29 & 54 & 59 & 69 & 50 \\
\hline \multirow{8}{*}{ Escolaridade } & \multirow{4}{*}{ Pai } & $1^{\mathrm{o}} \mathrm{g}$ & 3 & 12 & 5 & 26 & 17 & 1 \\
\hline & & $2^{\mathrm{o}} \mathrm{g}$ & 10 & 8 & 25 & 15 & 17 & 24 \\
\hline & & $3^{0} \mathrm{~g}$ & 44 & 47 & 46 & 33 & 30 & 35 \\
\hline & & Pós-g & 43 & 33 & 24 & 26 & 36 & 40 \\
\hline & \multirow{4}{*}{ Mãe } & $1^{o} \mathrm{~g}$ & 0 & 4 & 0 & 11 & 11 & 3 \\
\hline & & $2^{\underline{o}} \mathrm{~g}$ & 18 & 25 & 31 & 22 & 25 & 21 \\
\hline & & $3^{\circ} \mathrm{g}$ & 60 & 38 & 46 & 37 & 36 & 41 \\
\hline & & Pós-g & 22 & 33 & 23 & 30 & 28 & 35 \\
\hline \multirow{2}{*}{ Computador em casa } & \multicolumn{2}{|l|}{ Sim } & 94 & 75 & 90 & 74 & 69 & 96 \\
\hline & Não & & 6 & 25 & 10 & 26 & 31 & 4 \\
\hline
\end{tabular}

Nota 1: Total de alunos $(\mathrm{N})=318$. $\left(^{*}\right)$ Os cursos 3 e 6 são participantes do Promed.

Nota 2: Tabela preparada a partir de dados/respostas aos questionários aplicados. 
TABELA 3

Respostas consolidadas dos alunos do $6^{\circ}$ ano médico ao questionário de pesquisa

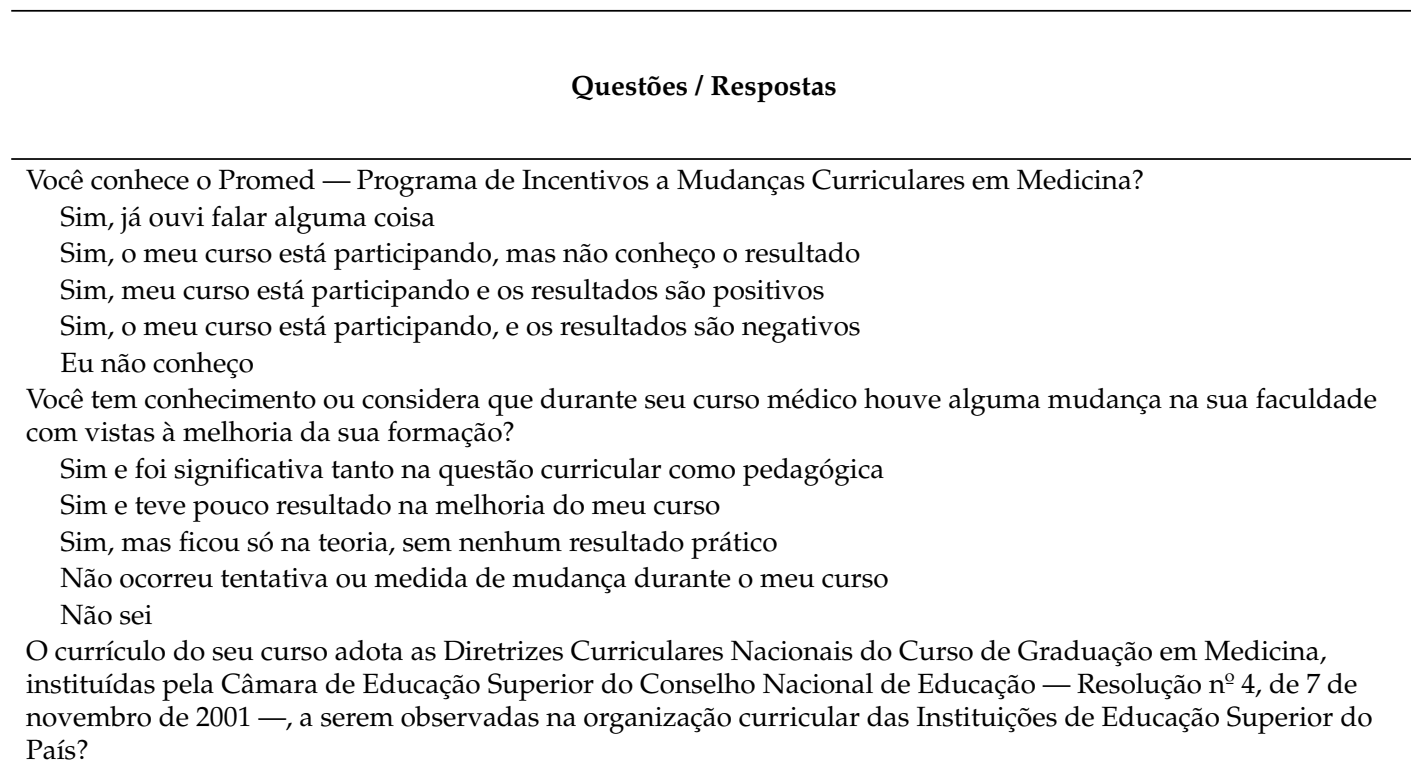

A maior parte delas

Somente uma parte

Não adota a resolução

Não sei

Outro

Seu curso discute a política de saúde e a relação formação/serviços de saúde?

Durante todo o curso, desde o primeiro ano

Esporadicamente durante uma ou outra disciplina

Somente no último ano

Só discute nos congressos, ficando a cargo dos alunos

Outro

O seu curso médico utiliza quais cenários como locais de prática para ensino e estágios?

Unidades básicas de saúde e hospitais do SUS, seguindo preceitos de regionalização e hierarquização de ações, de referência e c/referência

Hospital, serviços ambulatoriais da sua instituição, sem referência e c/referência

Somente hospitais de especialidades

Não existe sistematização definida

Outro

O seu curso médico procura estabelecer relação e participação comunitária, envolvendo instituições

populares?

Regularmente, existindo articulação ensino/problemas comunitários

É promovida eventualmente em problemas focais, dependendo da disciplina ou docente

Somente quando a comunidade procura

Não é promovida nem procura promover

Outro

\begin{tabular}{cccc}
$\begin{array}{c}\text { Alunos } \\
\text { Promed }\end{array}$ & \multicolumn{2}{c}{$\begin{array}{c}\text { Alunos não } \\
\text { Promed }\end{array}$} \\
N= 159 & \multicolumn{2}{c}{ N= 159 } \\
no & \% & n & \% \\
\hline & & & \\
36 & 22,7 & 31 & 19,5 \\
49 & 30,8 & 8 & 5,0 \\
32 & 20,1 & 0 & 0 \\
24 & 15,1 & 4 & 2,5 \\
18 & 11,3 & 116 & 73,0
\end{tabular}

Considerando as diversas tipologias de médico, ao final do seu curso, você pretende ser com maior ênfase que tipo de médico?

Médico generalista

Médico especialista

Pesquisador

Docente

Pesquisador-Docente

Seu curso tem como objetivo principal formar o médico, em nível de graduação, para:

Exercício imediato e integral da medicina nos aspectos preventivo, curativo, clínico geral e cirúrgico, pesquisa e articulação comunitária

Exercício geral da medicina, imediatamente, e obrigatoriamente buscar especialização — residência ou outra formação complementar

\begin{tabular}{|c|c|c|c|}
\hline 36 & 22,7 & 76 & 47,8 \\
\hline 28 & 17,6 & 20 & 12,6 \\
\hline 5 & 3,1 & 4 & 2,5 \\
\hline 82 & 51,6 & 57 & 35,9 \\
\hline 8 & 5,0 & 2 & 1,2 \\
\hline 21 & 13,2 & 33 & 20,8 \\
\hline 98 & 61,6 & 105 & 66,0 \\
\hline 3 & 1,9 & 2 & 1,2 \\
\hline 33 & 20,8 & 12 & 7,6 \\
\hline 4 & 2,5 & 7 & 4,4 \\
\hline 89 & 56,0 & 108 & 68,0 \\
\hline 54 & 34,0 & 37 & 23,3 \\
\hline 8 & 5,0 & 8 & 5,0 \\
\hline 5 & 3,1 & 5 & 3,1 \\
\hline 3 & 1,9 & 1 & 0,6 \\
\hline 30 & 18,9 & 76 & 47,8 \\
\hline 94 & 59,1 & 55 & 34,6 \\
\hline 18 & 11,3 & 6 & 3,8 \\
\hline 13 & 8,2 & 18 & 11,3 \\
\hline 4 & 2,5 & 4 & 2,5 \\
\hline 22 & 13,8 & 40 & 25,1 \\
\hline 22 & 76,8 & 103 & 64,8 \\
\hline 4 & 2,5 & 6 & 3,8 \\
\hline 6 & 3,8 & 3 & 1,9 \\
\hline 5 & 3,1 & 7 & 4,4 \\
\hline 7 & 4,4 & 68 & 42,8 \\
\hline 69 & 43,4 & 57 & 35,8 \\
\hline
\end{tabular}


TABELA 3

Respostas consolidadas dos alunos do 6ํano médico ao questionário de pesquisa (cont.)

\begin{tabular}{|c|c|c|c|c|}
\hline \multirow[t]{2}{*}{ Questões / Respostas } & \multicolumn{2}{|c|}{$\begin{array}{l}\text { Alunos } \\
\text { Promed } \\
\mathrm{N}=159\end{array}$} & \multicolumn{2}{|c|}{$\begin{array}{c}\text { Alunos não } \\
\text { Promed } \\
N=159\end{array}$} \\
\hline & no & $\%$ & no & $\%$ \\
\hline Atuar na atenção básica de saúde imediatamente, mas buscar formação complementar & 55 & 34,6 & 27 & 17,0 \\
\hline Buscar obrigatoriamente, imediata formação complementar & 25 & 15,7 & 7 & 4,4 \\
\hline Não sei & 3 & 1,9 & 0 & 0 \\
\hline \multicolumn{5}{|l|}{ Concluída sua graduação, você gostaria e começaria a trabalhar em que área? } \\
\hline Serviço geral de saúde, como o PSF — Programa Saúde da Família & 27 & 17,0 & 30 & 18,9 \\
\hline Serviços especializados em Clínica & 64 & 40,2 & 67 & 42,1 \\
\hline Serviços especializados em Cirurgia & 51 & 32,1 & 44 & 27,7 \\
\hline Serviço geral de saúde em articulação com ensino e pesquisa científica & 12 & 7,6 & 8 & 5,0 \\
\hline Outro & 5 & 3,1 & 10 & 6,3 \\
\hline \multicolumn{5}{|l|}{ Na sua atuação como médico, que tipo de serviço/vínculo você tende a escolher? } \\
\hline Em serviços públicos de assistência médica & 17 & 10,7 & 26 & 16,4 \\
\hline Em serviços públicos de gestão e administração de saúde & 4 & 2,5 & 8 & 5,0 \\
\hline Em serviços públicos de ensino & 15 & 9,4 & 5 & 3,1 \\
\hline Em medicina especificamente privada-particular & 22 & 13,8 & 16 & 10,1 \\
\hline Em medicina privada-particular e medicina pública ao mesmo tempo & 101 & 63,6 & 104 & 65,4 \\
\hline
\end{tabular}

Nesse caso em particular, esses dois estudantes apresentam concepções muito díspares, embora sejam alunos da mesma escola e estejam envolvidos pelo mesmo ambiente acadêmico, bem como submetidos aos mesmos processos educacionais e ao mesmo currículo. Isto nos leva a supor, como dito antes, que existe um conjunto de fatores que influenciam o processo de mudança na educação médica, bem como a compreensão e resposta dos estudantes.

Por um lado, isto reforça a idéia de complexidade da formação médica e de seu processo de mudança e, por outro, dá a impressão de que ainda é muito pouco conhecido o resultado das diferentes propostas de mudanças relacionadas ao ensino médico, o que carece ser mais bem estudado, avaliado e difundido.

Em virtude da mudança no Governo Federal, em 2003, novas políticas entraram na agenda do MS com maior prioridade. Embora os princípios dessas políticas não representassem conflitos com os do Promed ${ }^{22}$, após três anos de desenvolvimento dos 19 projetos do Promed, poucas têm sido as oportunidades de interação das escolas envolvidas, e a profundidade e relevância das mudanças diferem bastante entre as instituições participantes do programa, segundo avaliações individuais em boa parte das instituições ${ }^{22}$. Essa constatação foi também confirmada nas três oportunidades em que o Promed foi alvo de discussão coletiva: a oficina no Congresso da Rede Unida em Belo Horizonte ${ }^{30}$, em meados de 2005; o Seminário de Avaliação do Promed, realizado pelo Ministério da Saúde, em setembro de 2005, na cidade do Rio de Janeiro ${ }^{31}$; e outra Oficina Nacio- nal, no $43^{\circ}$ Congresso Brasileiro da Abem, realizado em Natal (RN), em outubro de 2005, de que participaram ao menos um docente, um discente e um gestor de cada projeto ${ }^{32}$.

No "Seminário de Avaliação do Promed", no Rio de Janeiro ${ }^{31}$, com participação de grande representatividade de todos os segmentos envolvidos no programa, parece que o consenso de maior importância é o de que faltou acompanhamento e avaliação por parte do Ministério da Saúde, considerado, também, fator fundamental para o fraco desempenho dos projetos. Observa-se assim, que, embora estivesse determinada na Portaria conjunta MEC-MS no 610/2002 ${ }^{33}$, a Comissão Nacional de Acompanhamento do Promed não foi efetivada ${ }^{31}$.

Nas diferentes oportunidades de discussão do Promed, portanto, aliada a uma ação dirigida à maior motivação, sensibilização e envolvimento de docentes ${ }^{31}$, foi uma constante a proposta de organizar um modelo de acompanhamento, avaliação e apoio ao programa. Seguramente, essa medida seria válida para qualquer iniciativa de indução e/ou adoção das Diretrizes Curriculares Nacionais, o que foi confirmado e ressaltado pelos sujeitos entrevistados nesse trabalho: "uma avaliação indutora e fomentadora" (docente).

A integração ensino-serviço também foi ponto crítico, conforme os entrevistados. Mesmo ao ressaltar alguns resultados importantes alcançados pelas escolas, o destaque maior era para as principais dificuldades enfrentadas, entre as quais:

- "Pouca sensibilidade dos docentes para as mudanças" (dirigentes e consultores); 
- "Pouco avanço na integração escola-serviços, com dirigentes de escolas dizendo que 'o médico do serviço não está em condições de ensinar'";

- "Outras escolas, que trabalham com Aprendizagem Baseada em Problemas - ABP (ou PBL - Problem Based Learning), (...) algumas não possuem sequer um profissional de saúde coletiva para colaborar na elaboração dos problemas" (consultor do projeto);

- "Os recursos financeiros, em algumas escolas, foram priorizados apenas para a contratação de consultores e pacotes de PBL" (dirigente recente de escola médica);

- "A IDA - Integração Docente-Assistencial ainda é somente um discurso em várias escolas" (gestor de serviço de saúde);

- "As mudanças de cenário de ensino do hospital para a rede encontram resistências de toda ordem, e muitos desses problemas nem sequer são discutidos" (membro da direção do Promed);

- "A falta de acompanhamento por parte do MS foi um fator fundamental para o pouco êxito do programa e, embora estivesse determinada por Portaria conjunta MEC/MS, a Comissão Nacional de Acompanhamento do Promed não foi levada a efeito" (esta questão obteve um amplo consenso entre os diferentes sujeitos entrevistados);

- "Bastante discutível é o fato de se falar muito em avanços nas escolas médicas, quando nenhuma delas colocou as implicações desses avanços para os serviços de saúde. É um problema ético sério a transformação da escola ser vista como possível de ser feita de forma isolada do serviço de saúde" (consultor do Promed);

- "A simples utilização da rede de serviços de saúde é insuficiente e incoerente com a transformação, pois trabalha apenas na ótica do quantitativo e não da resolução e da qualidade do atendimento" (gestor de serviço de saúde).

Essas manifestações, em alguns pontos contraditórias e conflitantes, em parte guardam relação com o que apontara o relatório de avaliação do Promed ${ }^{31}$. De certa forma, expressam a imagem de mosaico que ainda predomina no complexo processo de mudanças (curriculares e outras) da educação médica.

De fato, um sinuoso e intenso movimento de construção de consenso tem se verificado na grande quantidade de estudos, autores e proposições, com diferentes evidências científicas, concordando com a avaliação de que há um processo contínuo de mudanças na educação médica, intensificado nas duas últimas décadas, e que precisa ser ampliado e consolidado por dentro do sistema e serviços de saúde, em sintonia com a regionalidade estrutural e epidemiológica, e comprometido com as necessidades e prioridades sanitárias da população.
Por outro lado, a interação com ações de promoção da saúde, participação social-comunitária, vigilância em saúde, etc., em articulação com a gestão local do sistema de saúde, que pode representar uma importante estratégia de formação em saúde integral, na lógica proposta pelo SUS, foi muito enfatizada no discurso de todos ao observarem que "a relação ensino-serviço ficou muito na ótica das escolas; faltou a presença do gestor da saúde no Promed" (consultor).

Por isso mesmo, provavelmente, essa questão foi modificada no novo programa de ampliação do incentivo às mudanças curriculares (Programa Nacional de Reorientação da Formação Profissional em Saúde - Pró-Saúde), anunciado e lançado pelo MS no final de 2005, sendo consenso a importância de se montar, concomitantemente, um modelo de apoio, acompanhamento e avaliação ${ }^{23}$.

\section{DISCUSSÃO}

A análise da formação do médico é relevante pela importância desse profissional no sistema de saúde e na sociedade, e, sobretudo, por sua inserção crescente nas equipes multidisciplinares, amplamente multiplicadas nos últimos anos pela estratégia Saúde da Família. Segundo Pierantoni et al.34, é consensual a incapacidade de adequação das instituições formadoras à velocidade com que se demandam novos perfis profissionais, tanto no referencial para atuação técnica específica quanto na introdução de concepções pedagógicas que desenvolvam habilidades para apreensão e aplicação crítica dessas novas técnicas.

O Promed constitui um dos diversos dispositivos de indução de mudanças curriculares, na medida em que, nos diversos cursos médicos, tais mudanças se iniciaram no começo dos anos 1990 e sua discussão foi estimulada por diferentes iniciativas e projetos ${ }^{35}$. Por outro lado, as mudanças parecem ocorrer numa velocidade muito menor do que a expectativa dos serviços de saúde e as necessidades de saúde da população.

As escolas médicas possuem diferentes histórias e variados contextos em educação médica. Em algumas, as mudanças se iniciaram no começo dos anos 1990, e sua discussão foi estimulada pelo Projeto UNI ${ }^{35}$. Outras ações indutoras de mudanças foram capitaneadas pelo processo de construção do SUS, mas foi o Promed que, em 2002, retomou o processo de discussão do curso de medicina em várias instituições no contexto das Diretrizes Curriculares Nacionais.

As concepções de medicina e os objetivos da formação médica parecem ter diferenças em cada uma das disciplinas e áreas da educação médica, o que prejudica o diálogo. Isto seria superado com a criação de um diálogo interdisciplinar 
que representaria maior integração teoria-prática, só possível numa integração ensino-serviços de saúde. Para lidar com a dualidade representada pela tecnificação da prática médica e pelo aumento crescente do agravo de problemas básicos de saúde da população, o médico deve estar apto a questionar seu papel diante de duas vertentes e a atuar na melhoria da qualidade de vida e reduzir os níveis de adoecer. Nesse sentido, a reformulação curricular nessa direção tem seus limites e resistências ${ }^{36}$.

Uma delas seria a preparação de professores identificados e comprometidos com o processo de mudanças. Segundo Abreu Neto et al. ${ }^{37}$, "em qualquer iniciativa de reformulação curricular, a capacitação docente é considerada fundamental para o sucesso do processo de implementação e sustentação das mudanças. Professores resistentes e indiferentes às mudanças dificultam o trabalho de reformulação curricular".

E se ainda preparam-se profissionais de saúde distanciados da realidade dos serviços de saúde e pouco comprometidos, até por desconhecimento, com o processo pelo qual está passando o modelo de atenção à saúde, avaliação e transformação passaram a ser temas prioritários e de interesse de diferentes instituições, tanto da área acadêmica como do setor de serviços ${ }^{35}$.

A interação de ações de promoção da saúde, participação social-comunitária, vigilância em saúde, etc., em articulação com a gestão local do sistema de saúde, representa uma importante estratégia de formação em saúde integral, na lógica proposta pelo SUS. Entretanto, a análise das respostas dos alunos nesta pesquisa e seus depoimentos sobre a relação e participação das organizações comunitárias e populares (na integração ensino-serviços de saúde), indica que esta é praticamente inexistente, o que pode significar que a importância da sociedade e de suas instâncias para o ensino médico é, por enquanto, uma realidade muito distante.

Embora carecendo de uma análise mais aprofundada, e de outras questões que pudessem ser formuladas ao conjunto de alunos dos cursos médicos - para melhor compreender as possíveis causas das diferenças observadas nas respostas do presente trabalho - , há uma tendência a considerar de pouco significado o fato de o "curso médico" ser participante ou não do Promed.

Pelo que se viu no grupo de cursos analisados, mesmo em temas que importam muito ao Sistema Único de Saúde - como a qualidade do ensino e do trabalho desses médicos quando formados, a relação entre ensino e serviços de saúde e o acesso aos cursos de Medicina ${ }^{38}$-, o Promed não pode ser considerado como influência decisiva para os resultados, de acordo com as respostas verificadas.
Considerando somente os cursos não participantes do Promed, estes apresentam diferenças entre si, o que era de se esperar em função de diversos fatores não pesquisados, como tempo de existência do curso, natureza jurídica e localização do curso em cidades menores, e mesmo a existência de outras iniciativas de mudanças visando à implantação das Diretrizes Curriculares Nacionais.

No entanto, essa e outras propostas de mudança reforçam o entendimento de que, no Brasil, ultimamente, nenhuma outra palavra tem sido tão associada à educação médica como a palavra mudança ${ }^{39}$. Além do mais, mesmo considerando que o Promed não foi totalmente implantado - sendo este um certo consenso observado nas entrevistas com docentes, dirigentes de escolas médicas e consultores do Promed -, observa-se que a discussão sobre mudanças curriculares e o debate desencadeado, além de ter produzido uma série de repercussões dentro de cada escola ${ }^{31}$ que precisam ser mais bem investigadas, possibilitou um desdobramento importante, sintetizado na criação do Pró-Saúde ${ }^{40}$.

Enquanto as universidades, segundo Chauí ${ }^{41}$, correspondem exatamente ao perfil da sociedade atual, pelas diversas formas de violência que se fazem presentes dentro delas ("desigualdades, favorecimentos, reforço de privilégios, burocracia, submissão aos padrões de produtividade neoliberais, silêncio diante da privatização do que é público..."), reafirmando sua tradição de autonomia, elas, na maioria dos casos, seguem sua lógica própria, o que, certamente, tem dificultado uma aproximação maior entre as IES e a sociedade, na discussão e encaminhamentos sobre a formação de profissionais de saúde, sobretudo a formação do médico ${ }^{31}$.

A universidade, de modo geral, tende a sofisticar seus serviços, enfatizando o ensino e a pesquisa em detrimento da atenção individual e comunitária. Barbosa et al. ${ }^{42}$ defendem que não se pode ensinar adequadamente medicina, e muito menos educar o médico, sem contar com um razoável serviço de saúde. Os serviços, por outro lado, em suas obrigações assistenciais, tendem a se fechar em si mesmos e a cair numa rotina tradicional. Chega-se, então, a uma continuada dissociação entre os serviços de educação e os serviços de saúde e vice-versa.

Entre as dificuldades que ainda representam obstáculos a superar, algumas significam importantes desafios ao desenvolvimento e aprimoramento desse processo de mudanças: a pouca tradição da direção de escolas médicas na elaboração e gestão de projetos inovadores; o estabelecimento de parcerias interinstitucionais e transetoriais; a reorientação da missão dos cursos de medicina para formar profissionais identificados com as necessidades da população; o maior entrosamento entre o mundo do ensino e a realidade dos serviços, numa in- 
tegração cotidiana e crescente entre ensino, serviços de saúde e comunidade; um continuado intercâmbio entre as diferentes experiências exitosas, como forma de contribuição e aceleração de incorporação de mudanças ${ }^{31}$.

Além do mais, falta um diagnóstico que expresse a visão de conjunto dos gestores, educadores e trabalhadores da saúde, tratando a questão de recursos humanos para a saúde (incluindo o médico) de forma coerente e articulada, e em âmbito nacional ${ }^{17}$.

No País, os cursos de medicina estão localizados em pouco mais de uma centena de municípios. Portanto, se ocorresse uma efetiva integração e articulação entre gestores municipais de saúde e as escolas médicas de seus respectivos municípios, seguramente isso possibilitaria maior aceleração no processo de mudanças, adequando melhor a formação e a correspondente utilização desses médicos nos sistemas locais e regionais de saúde.

A colaboração entre serviços, centros de pesquisa e universidades, associada à dupla militância do quadro de pesquisadores dos centros de pesquisa, possibilita real colaboração e complementaridade entre ambos os tipos de instituições ${ }^{43}$. Ressalte-se que este aspecto constitui um desafio fundamental na formação de pessoal para a saúde no contexto do SUS e praticamente não foi abordado nos diferentes momentos e oportunidades de discussão e avaliação do Promed e não consta entre seus resultados mais positivos, nem foi abordado nesta nossa pesquisa.

Assim, este campo continua em aberto e para seu estudo seria muito interessante associar mais aspectos relacionados à visão dos alunos sobre esse dinâmico processo de mudança da formação médica.

Verificando-se os pontos centrais que constituem desafios e que também representam perspectivas a serem consideradas para a continuidade e aprofundamento dessa discussão, diferentes aspectos ressaltados nos resultados deste trabalho podem suscitar outras contribuições para ampliar e acelerar o debate em torno dessa mudança na formação médica.

Mesmo o Promed representando uma tentativa oportuna e estratégica de discussão e adoção de mudanças na graduação de medicina e que se desdobrou em outra iniciativa mais ampla de mudanças (o Pró-Saúde), há necessidade de estudos para conhecer o impacto dessas mudanças na graduação do médico brasileiro, sintonizadas com as Diretrizes Curriculares Nacionais e voltadas para as necessidades de saúde da população. Esse pensamento deve nortear também qualquer iniciativa de avaliar outras propostas de incentivos a mudanças curriculares que vêm se passando nos cursos e escolas de medicina, mesmo sabendo-se que, de início, o interesse pela proposta de mudança se deu, e ainda se dá, muito em função do incentivo financeiro correspondente.
A importância da proposta do Promed e sua potencialidade de promover transformações, como de qualquer outro dispositivo com essa pretensão, exigem atenção e cuidados bastante detalhados quanto à sua estruturação, resultados e desdobramentos, que possam ser observados num processo contínuo de avaliação e acompanhamento.

As iniciativas direcionadas para a mudança tendo por base as Diretrizes Curriculares Nacionais, aumentadas em volume e em diferentes regiões, ainda carecem, contudo, segundo nossos levantamentos e respostas dos alunos à nossa pesquisa, de mais envolvimento dos docentes e dos discentes, sendo que estes, segundo suas respostas, têm dificuldade de reconhecer resultados significativos nessas iniciativas.

\section{CONCLUSÕES}

Parece evidente um contrapasso entre a ênfase dada pela maioria das escolas médicas pesquisadas e o perfil do médico que os serviços de saúde e a comunidade precisam. Apesar de estar ampliando o número de cursos médicos que têm discutido mudanças, somente uma minoria desses cursos tem implantado disciplinas e/ou programas de iniciação científica, bem como promovido a participação comunitária/popular, duas questões absolutamente fundamentais para a formação médica no atual contexto do sistema de saúde.

Se, por um lado, existem experiências inovadoras desde a década de 1960 (UnB, USP, UFMG), espalhadas em diferentes regiões do território brasileiro, que representam perspectivas promissoras de efetiva modificação do quadro de ensino-aprendizagem e de maior adequação às necessidades dos serviços e sistemas de saúde, da formação e qualificação dos profissionais de saúde, em geral, e do médico em especial, por outro lado, dirigentes dos serviços de saúde (especialmente os do nível local) e responsáveis pelas instituições de ensino médico precisam em conjunto criar condições concretas de exercícios e práticas promotores dessa mudança.

Uma medida imediata, diante desse contexto, é o estabelecimento de parcerias, protocolos de cooperação sistemática e auto-sustentável entre gestores do SUS e as escolas médicas no sentido de buscar a integração da academia com os serviços de saúde, visando à formação de profissionais adequados e qualificados para atender às necessidades de saúde da população brasileira. Há condições concretas, vontades, oportunidades e recursos para estas propostas se viabilizarem.

\section{Contribuição de cada um dos autores na realização deste trabalho:}

Neilton Araujo de Oliveira: levantamento bibliográfico e documental, trabalho de campo (questionários, entrevistas, 
observação participante), elaboração da proposta básica do texto, tabulação e análise de dados, discussão dos resultados, revisão compartilhada e redação do texto final; Rosane Moreira Silva de Meirelles: colaborou na discussão dos resultados e na redação do texto; Geraldo Cunha Cury: colaborou na redação do texto; Luiz Anastácio Alves: colaboração no levantamento bibliográfico, aprimoramento e aprovação do desenho do trabalho, discussão dos resultados, revisão compartilhada, redação do texto final.

\section{REFERÊNCIAS}

1. Arruda BKG, org. A educação profissional em saúde e a realidade social. Recife: Instituto Materno Infantil de Pernambuco (IMIP), Ministério da Saúde; 2001.

2. Brasil. Constituição da República Federativa do Brasil. Brasília: Senado Federal; 1988.

3. Brasil. Ministério da Saúde. Conselho Nacional de Saúde O Desenvolvimento do Sistema Único de Saúde: avanços, desafios e reafirmação dos seus princípios e diretrizes / MS, Conselho Nacional de Saúde. Brasília: Ministério da Saúde; 2002.

4. Stella RCR. Conquistas e Desafios [editorial]. Rev. Bras. Educ. Méd. 2001; 25:2.

5. Paim JS. Recursos Humanos em Saúde no Brasil: problemas crônicos e desafios agudos. São Paulo: Facudade de Saúde Pública; 1994.

6. Brotherton SE, Rochey PH, Etzel SI. American Medical Association. US Graduate Medical Education, 2003-2004. JAMA 2004; 292:1032-37.

7. Lempp H, Seale C. The hidden curriculum in undergraduate medical education: qualitative study of medical students' perceptions of teaching. BMJ 2004; 329:770-73.

8. Smits PBA, Verbeek JHAM, Nauta MCE, Tem Cate ThJ, Metz JCM, Van Dijk FJH. Factors predictive of successful learning in postgraduate medical education. Med. Educ. 2004; 38:848-51.

9. Ferguson E, James D, Madeley L. Factors associated with success in medical school: systematic review of the literature. BMJ 2002 324:952-57.

10. Organização Pan-Americana de Saúde. Anais do Encontro Continental de Educação Médica. Montevideo: OPS/OMS; 1997.

11. World Health Organization. Changing medical education and practice: an agenda for action. Genebra; 1991.

12. Conferência Internacional de Alma-Ata. Cuidados primários de saúde. Brasil: Unicef, 1978. (Relatório).

13. Anais do XLII Congresso Brasileiro de Educação Médica; Vitória-ES; 2004 - Anais do XLIII Congresso Brasileiro de
Educação Médica. Natal-RN; 2005 — Anais do XLIV Congresso Brasileiro de Educação Médica. Gramado-RS; 2006.

14. Conselho Nacional de Secretários Municipais de Saúde. XVII Congresso Nacional dos Secretários Municipais de Saúde. Brasília: Conasems; 2001. [capturado 6 maio 2006]. Disponível em: http:/ / www.conasems.com.br. (Relatório Final)

15. Conselho Nacional de Saúde. 11ª Conferência Nacional de Saúde. Brasília: Ministério da Saúde; 2001 — 12ª Conferência Nacional de Saúde - Propostas e Diretrizes do Ministério da Saúde para os Eixos Temáticos. Brasília: Ministério da Saúde; 2003.

16. Ministério da Educação. Coordenação de Aperfeiçoamento Pessoal de Nível Superior. Banco de teses. [online] Brasília [capturado 11 jun. 2006]. Disponível em:

http://www.capes.gov.br/servicos/bancoteses.html

17. Stella RCR. Desafios e Estratégias para Interiorização do Trabalho em Saúde. In: Política de Recursos Humanos em Saúde: Seminário Internacional / Brasil. Brasília: Ministério da Saúde; 2002.

18. Oliveira NA. Medicina e Saúde na Nova Universidade Federal do Tocantins. Anais XLII Congresso Brasileiro de Educação Médica; 2004 nov. 20-24; Vitória, Brasil. Rio de Janeiro: Associação Brasileira de Educação Médica; 2004.

19. Oliveira NA. A espetacularidade do "Saúde da Família" e o rumo norte do SUS. Saúde \& Cidade. Rio de Janeiro: 1999; p.12-3.

20. Comissão Interinstitucional Nacional de Avaliação do Ensino Médico. Avaliação do ensino médico no Brasil: relatório geral 1991-1997. Brasília; 1997.

21. Almeida M, orgs. Diretrizes Curriculares Nacionais para os Cursos Universitários da Área da Saúde. Londrina: Rede Unida; 2003.

22. Brasil. Ministério da Saúde. Promed-Programa de Incentivo às Mudanças Curriculares para as Escolas Médicas. Brasília: Ministério da Saúde; 2002.

23. Brasil. Ministério da Saúde. Pró-Saúde-Programa Nacional de Reorientação da Formação Profissional em Saúde. [online] Brasília: Ministério da Saúde; 2005. [capturado 8 out. 2006]. Disponível em: http:/ / www.saude.gov.br/sgtes.

24. Koifman L. O Ensino Médico no Brasil e na Argentina: uma abordagem comparativa. Rio de Janeiro; 2002. Doutorado (Tese) — Escola Nacional de Saúde Pública.

25. Brasil. Poder Legislativo. Lei Orgânica da Saúde no 8080 , de 19 de setembro de 1990. Dispõe sobre as condições para a promoção, proteção e recuperação da saúde, a organização e o funcionamento dos serviços correspondentes e da outras providencias. Diário Oficial [da] República Federa- 
tiva do Brasil. Brasília, DF, 20 set. 1990, Seção 1, p. 18.055. Acesso 06/05/06 e disponível em: http://www.saúde. gov.br/doc/lei 8080.htm.

26. Campos GWS. Análise crítica sobre especialidades médicas e estratégias para integrá-las ao SUS. Cad. Saúde Pública Jan 1997; vol.13(n.1):141-144. ISSN 0102-311X.

27. Batista NA, Silva SHS. O Professor de Medicina. São Paulo: Edições Loyola, 2ª ed, 2001

28. Minayo MCS (org). Pesquisa social: teoria, método e criatividade. Petrópolis, RJ: Vozes, 21a edição, 2002.

29. Bardin L. Análise de conteúdo. Lisboa: Edições 70; $1977 .$.

30. VI Congresso Nacional da Rede Unida. Belo Horizonte, 2005. [capturado 11 jun 2006]. Disponível em: http:// www.ufmg.br/redeunida/oficinas/OFICINA \%2044\%20\%20Com\%20Logo\%5B1\%5D.doc.

31. Brasil. Ministério da Saúde. Relatório do Seminário de Avaliação do Promed. Brasília: Ministério da Saúde; 2005.

32. XLIII Congresso Brasileiro de Educação Médica; 2005 set. 26-29; Natal; 2005. Rio de Janeiro: Associação Brasileira de Educação Médica; 2005 [capturado 12 jun. 2006]. Disponívelem:http://www.abem-educmed.org.br/cobem/2005/ natal_ventureli.pdf

33. Brasil. DOU-Diário Oficial da União. 01/04/2002, seção 1, p 75, imprensa nacional; 2002.

34. Pierantoni CR, Viana ALA, Tavares RAW, Rodrigues, SH, Belisário SA \& França T. Avaliação do curso de atualização em gestão municipal na área de saúde: uma proposta de metodologia. In: Falcão A, Santos Neto PM, Costa PS, Belisário SA orgs. Observatório de Recursos Humanos em Saúde no Brasil: Estudos e Análises. Rio de Janeiro: Editora Fiocruz; 2003.

35. Feuerwerker LCM, Llanos M. Educação dos profissionais de saúde na América Latina: teoria e prática de um movimento de mudança. São Paulo: Hucitec; Buenos Aires: Lugar Editoria; Londrina: Editora Uel, 1999.
36. Koifman L. O modelo biomédico e a reformulação do currículo médico da Universidade Federal Fluminense. História, Ciências, Saúde 2001; 8(1): 49-70.

37. Abreu Neto IP, Lima Filho OS, Silva LEC, Costa NMSC. Percepção dos Professores sobre o Novo Currículo de Graduação da Faculdade de Medicina da UFG Implantado em 2003. Rev. brs educ méd 2006;30(3):154 -60.

38. Petroianu A, Santos BMR, Gonçalves RM. Performance by Medical Students in Testing Specific Knowledge. Rev. Bras. Educ. Méd. 2004; 28:128-32.

39. Facchini LA., Piccini RX, Santos RC. Aspectos históricos e conceituais em educação médica. [online]. 1998. [capturado 23 dez. 2006]. Disponível em http://www.unb.br/fs / pr33.htm.

40. Brasil. Ministério da Saúde. Pró-saúde: programa nacional de reorientação da formação profissional em saúde. Ministério da Saúde, Ministério da Educação. Brasília: Ministério da Saúde; 2005.

41. Chauí M. Ideologia neoliberal e universidade. In: Oliveira F, Paoli MC orgs. Os sentidos da democracia: políticas do dissenso e hegemonia global. Petrópolis: Vozes; 1999.

42. Barbosa FS, Carvalho AG, Lavor CH, Santana JFNP. Atenção à saúde e educação médica: uma experiência e uma proposição. Educación médica y salud. 1977; 11(1): 26-40.

43. Conh A, Vigevani T. A produção do conhecimento e os centros de pesquisa: a trajetória do Cedec. Perspec 2002;16(3): $42-7$.

\section{CONFLITOS DE INTERESSE}

Declarou não haver

\section{ENDEREÇO PARA CORRESPONDÊNCIA}

110 Sul, Alameda 21- no 56-58

Palmas - Tocantins

CEP. 77110-310

E-mail: neilton@uft.edu.br 